\title{
Differential impacts of copepods and cladocerans on lake seston, and resulting effects on zooplankton growth
}

\author{
Claes Becker ${ }^{1, *}$, Heidrun Feuchtmayr ${ }^{1}$, Daniela Brepohl ${ }^{2}$, Barbara Santer ${ }^{1} \&$ Maarten Boersma $^{3}$ \\ ${ }^{1}$ Max-Planck-Institut für Limnologie, Postfach 165, 24302 Plön, Germany \\ ${ }^{2}$ Institut für Meereskunde, Experimentelle Ökologie, Düsternbrooker Weg 20, 24105 Kiel, Germany \\ ${ }^{3}$ Alfred-Wegener-Institut für Polar und Meeresforschung, Biologische Anstalt Helgoland, Postfach 180, 27483 Helgoland, \\ Germany \\ (*Author for correspondence: Tel.: +49-4522-763231; E-mail: becker@mpil-ploen.mpg.de)
}

Key words: Daphnia, copepod, stoichiometry, homeostasis, feeding, growth, PUFA

\begin{abstract}
In an enclosure study in Schöhsee, a small mesotrophic lake in Northern Germany, the impact of copepods and daphniids on the seston community was studied. In general, these two guilds differ in their feeding behaviour. Copepods actively select their food, with a preference for larger particles, whereas most cladocerans are unselective filter-feeders. In this study we investigate how the impact of the two different grazers affects zooplankton growth. We combine results obtained in the laboratory with results measured in situ in the enclosures. Copepods and cladocerans were cultured on seston from enclosures that were inhabited by density gradients of copepods or daphniids. We observed that Daphnia grew faster on seston that was pre-handled by copepods than on seston that was pre-handled by daphniids, and that somatic growth decreased with increasing densities of daphniids in the enclosures. In contrast, we observed no differences in development rates for copepods grown on the different media. The population growth rates of Daphnia in the Daphnia treatments were determined in the enclosures. Growth differences in both somaticand population growth of Daphnia were correlated to food quality aspects of the seston. In the laboratory we found that Daphnia growth was correlated with several fatty acids. The strongest regression was with the concentration of 20:4 $33\left(r^{2}=0.37\right)$. This particular fatty acid also showed the highest correlation with growth after normalisation of the fatty acids to the carbon content of the enclosures $\left(r^{2}=0.33\right)$. On the other hand, in the enclosure the population growth correlated most to the particulate nitrogen content $\left(r^{2}=0.78\right)$ and only to the $\mathrm{N}: \mathrm{C}$ ratio, when normalised to carbon $\left(r^{2}=0.51\right)$.
\end{abstract}

\section{Introduction}

Ever since Hutchinson (1961) formulated his paradox of the plankton and Tilman (1982) investigated the conditions necessary for the coexistence of species in a certain habitat, many researchers have investigated the interactions between planktonic organisms. This was often done with phytoplankton (Flöder \& Sommer, 1999; Huisman \& Weissing, 1999; Interlandi \& Kilham, 2001), most likely since resources are easily defined for phyto- plankton. The interactions between freshwater zooplankters have also been investigated in some detail, especially the (competitive) interactions between members of one of the most obvious taxa in freshwater environments, the Cladocera (DeMott \& Kerfoot, 1982; Matveev, 1983; Bengtsson, 1986; Vanni, 1986; Hessen, 1990; Boersma, 1995). Interestingly, the potential interactions between copepods and cladocerans have received much less 
attention, even though members of these guilds cooccur in many freshwater bodies. Whereas feeding of cladocerans, especially daphniids is well studied and these animals are normally classified as herbivorous, the nutrition of copepods is less clear. Adult cyclopoid copepods are commonly accepted to be predaceous, and as a result the predator-prey interaction between cyclopoid copepods and cladocerans has been studied in some detail (e.g. Kerfoot, 1977; Gliwicz, 1994; Gliwicz \& Umana, 1994). Nevertheless, not all of the copepod species and stages are predaceous, and many feed on protists and algae (Santer \& van den Bosch, 1994; Jürgens et al., 1996), but with a different feeding mode compared to herbivorous cladocerans. Copepods actively select their food, while most cladocerans are filter-feeders and do not discriminate between food particles (DeMott, 1986; Butler et al., 1989). Food selection by copepods is very sensitive and copepods can even distinguish (probably via chemoreception) between food particles of different nutritional status (Cowles et al., 1988) or toxicity (DeMott \& Moxter, 1991). Members of the genus Daphnia, the most important cladoceran genus in many temperate lakes, on the other hand, can only reject already captured food particles. This procedure is ineffective since feeding is interrupted until the clogging particle is removed (Lampert, 1987). Moreover, edible particles may be removed together with the rejected particles. Even though daphniids lack the possibility to actively select single food particles, they are able to localise patches with high food abundance (e.g. Cuddington \& McCauley, 1994; Jensen et al., 2001). As a result of their high feeding efficiency and their parthenogenetic mode of reproduction, with the resulting rapid potential of population growth they can rapidly exploit such food sources. In contrast, copepods go through several naupliar and copepodite stages, show low feeding efficiency (Santer \& van den Bosch, 1994), and rely on sexual reproduction. This implies that copepods are probably much less effective in depleting their resources. In short, as a result of the differences in feeding preferences and feeding modes and life cycles, copepods and cladocerans affect the seston community to a different extent (Sommer et al., 2001).

A few studies have shown that in ecosystems where herbivorous copepods are dominant, smal- ler sized phytoplankton species prevail, since larger particles are preferred by the larger copepodite stages and the adults. On the other hand, in a Daphnia dominated system the larger phytoplankton species are most abundant, since these species are outside the range filtered by Daphnia (Rothhaupt, 1997; Sommer et al., 2001). Moreover, Rothhaupt (1997) also showed that not only differences in the phytoplankton species composition could be observed, but also that the main herbivores caused differences in the nutrient dynamics. Whereas daphniids, with their high requirement for phosphorus, drove the algae to $\mathrm{P}$ limitation, in systems with copepods both $\mathrm{N}$ and $\mathrm{P}$ remained limiting. This effect is explained by the fact that the different grazers differ in their nutrient content and requirements. Daphniids generally have a higher requirement for phosphorus than copepods, with molar C:P ratios of around 30 and 135 respectively (Hessen \& Lyche, 1991; but see also Villar-Argaiz \& Sterner, 2002). Therefore, daphniids will retain much of the phosphorus and thus cause P-limitation in the phytoplankton. Most likely, as a result of shifts in phytoplankton composition and nutrient availability when exposed to different herbivores also other aspects of the seston will change, such as the biochemical composition (fatty acids, amino acids). However, as still very little is known about the requirements of copepods and cladocerans for such substances it is not possible to predict the direction of these changes, nor the consequences.

In short, there are many differences between the cladocerans and copepods, which can cause differential effects on the food web. As in many lakes members of these taxa co-exist, it is very interesting to study how they affect each other. Possibly, given their different mode of feeding, the competition between the taxa will not be large, and they fill different niches. If this is the case, it could well be that by removing a food source, which is not readily available to the other crustaceans in the system, but competes with these food sources for nutrients, the presence of one taxon could actually be of benefit to the other one. This we aim to investigate in this paper. Moreover, by correlating several seston characteristics with the growth and reproduction of the organisms we aim to identify the parameters of the food that play a role in this interaction. 


\section{Materials and methods}

This work was done as a part of a larger project, in which we aim to determine the impact of different herbivorous zooplankton on the food web. These effects are studied in detail on various levels ranging from bacteria to zooplankton (Sommer et al., 2001, 2003). In this paper we focus on the interaction between the seston community and the zooplankton growth.

A mesocosm experiment was conducted in mesotrophic Schöhsee in May 2001. 24 mesocosms (2.2 m long; $\left.1.6 \mathrm{~m}^{3}\right)$ were installed and filled with lake water, which was sieved through $55 \mu \mathrm{m}$ plankton gauze in order to remove mesozooplankton, and enriched with a logarithmic density gradient of different zooplankton species (for more information about the set-up see Sommer et al., 2001). The Daphnia density gradient was obtained by adding a clone of Daphnia galeata $x$ hyalina originally isolated from lake Schöhsee. For the copepod density gradient we used wild-caught zooplankton samples $(<250 \mu \mathrm{m})$ from Schöhsee, which were dominated by the calanoid copepods Eudiaptomus gracilis and Eudiaptomus graciloides and the cyclopoid copepods Mesocyclops leuckarti, Diacyclops bicuspidatus and Thermocyclops oithonoides. To remove the cladocerans from the samples, the water was vigorously bubbled with air for several hours, during which the floating cladocerans were repeatedly removed from the water surface. The inoculated copepod samples contained calanoid and cyclopoid copepods in a ratio of about $1: 1$. The copepod enclosures were enriched with copepods to obtain densities of 5, 10, 20, 40 and 80 individuals per litre. The Daphnia density gradient was inoculated to achieve the enclosure densities of $1.25,2.5,5,10$ and 20 individuals per litre. We had a mixed treatment, which was inoculated with 20 copepods and 5 daphniids per litre. There was also a control treatment that did not receive any zooplankton. All together we had 12 different treatments that all were replicated yielding 24 enclosures. Different numbers were used for copepods and cladocerans to obtain similar biomass rather than similar numbers in the enclosures (see Sommer et al., 2001). Water and zooplankton samples were taken every three to four days. In order to avoid sampling errors due to aggregation or sedimentation, the enclosures were thoroughly mixed with a Secchi disk before sampling. Filtrates of the water samples were analysed for nutrient $(\mathrm{C}: \mathrm{N}: \mathrm{P})$ and fatty acid composition. For seston $\mathrm{C}: \mathrm{N}: \mathrm{P}$ analyses, pre-filtered $(100 \mu \mathrm{m})$ water was collected on pre-combusted and, for phosphorus, acid washed, GF/F glass fibre micro filters and dried overnight at $60{ }^{\circ} \mathrm{C}$. Total nitrogen and carbon was measured using a FISONS® NA2000 elemental analyser, total phosphorus was determined by an alkaline persulphate oxidation (Grasshoff et al., 1983). Fatty acid spectra were also established for the seston in the enclosures, and at the end of the experiment also for the zooplankton. For the seston analyses we filtered $2-51$ on a GF/C filter that was subsequently stored under $\mathrm{N}_{2}$ gas at $-70{ }^{\circ} \mathrm{C}$ until further processing. For the analysis of the zooplankton, we collected animals (1-5 mg dry weight) from each enclosure at the end of the experiment, sorted them, and stored them under nitrogen. Fatty acids were extracted, esterified and analysed on a gas chromatograph according to Wiltshire et al. (2000). To quantify the fatty acid content we used an internal standard of odd-chained fatty acid methyl esters (13:0-21:0; Restek).

Zooplankton samples were taken with two vertical tows through the entire water column of the enclosure with a $55 \mu \mathrm{m}$ mesh size plankton net. The samples were fixed with ethanol. The zooplankton was identified and counted under a dissecting stereomicroscope. From those counts, we calculated the population growth rates of the zooplankters in the enclosures.

During this mesocosm study we conducted two laboratory experiments, in order to determine how the differences between copepod and Daphnia treatments affect growth and development of different zooplankton taxa. The two laboratory experiments were conducted during the periods 912 and 13-18 days after the start of the enclosures. In both experiments, 2.51 water was taken daily from both replicate bags from the enclosure treatments. In the first experiment we used water from the following treatments: control, mixed, copepod 10 and 80, Daphnia 10 and 20 (number corresponds to individuals per litre). The second experiment was conducted with water from control, mixed, copepod 5 and 20, Daphnia 1.25 and 5. The water from each treatment was mixed and sieved over a $100 \mu \mathrm{m}$ gauze to remove most of the mesozooplankton. The water was kept dark and 
gently stirred to prevent sedimentation. The laboratory temperature during both experiments was $16{ }^{\circ} \mathrm{C}$, which was the ambient temperature of the enclosures. For the laboratory experiment we used Daphnia magna, our standard test clone to test for food quality effects (Boersma, 2000). This clone was originally collected from a pond in Frankfurt and has been kept at the Max Planck Institute for Limnology for many years. The experimental mothers were fed Scenedesmus obliquus ( $1 \mathrm{mgC}^{-1}$ ) every day and the medium was changed twice weekly. Third brood offspring were collected from these mothers within $24 \mathrm{~h}$ after birth and randomly divided in $120 \mathrm{ml}$ flowthrough vessels, filled with water from the separate enclosure treatments, with a flow rate of $11 \mathrm{day}^{-1}$. Each vessel contained five animals and we had five replicates per treatment. Six juveniles were randomly selected and placed on an aluminium weighing boat to determine initial dry mass. After 3 or 5 days for the first and the second experiment respectively, the experiment was terminated. All animals from each experimental vessel were harvested, and put together on an aluminium boat. These were then dried at $60{ }^{\circ} \mathrm{C}$ overnight and weighed to the nearest $0.1 \mu \mathrm{g}$. The increase in Daphnia body biomass per day (somatic growth rate) was computed for each treatment.

For the copepods, we determined the development of calanoid juveniles that were caught from Lake Schöhsee on the start day of the experiment. The copepodites were anaesthetised with carbonated water and 20 copepodites were randomly pooled together and placed in $120 \mathrm{ml}$ flow-through vessels in a quadruplicate set-up. Four vessels were additionally filled and harvested immediately to establish initial stage distributions, the other vessels were emptied after five days. All animals were fixed with $4 \%$ formaldehyde solution and the copepodite stages were determined under a stereomicroscope. The average copepodite stage was calculated with a start and an end value.

Growth of Daphnia was tested in both experiments, whereas the development of the copepods was tested in the second experiment only. Since copepods have a longer generation time compared to daphniids they should be given more time to show a noticeable response, therefore the second experiment lasted 5 days instead of 3 days, as was the case with the first experiment.
The results from all growth studies (somatic and population) were correlated with the seston parameters in the enclosures in order to determine potential limitations. For these computations we used the average values of the seston parameters over the duration of the laboratory experiments.

\section{Results}

In the first growth experiment, conducted 9 days after the start of the enclosure experiment, there were only small differences in Daphnia growth when fed seston from different enclosures. However, there was a tendency that the daphniids grew faster on seston pre-handled by copepods compared to Daphnia pre-handled seston (ANOVA; $p=0.06$; Fig. 1, top panel). In the second experi-
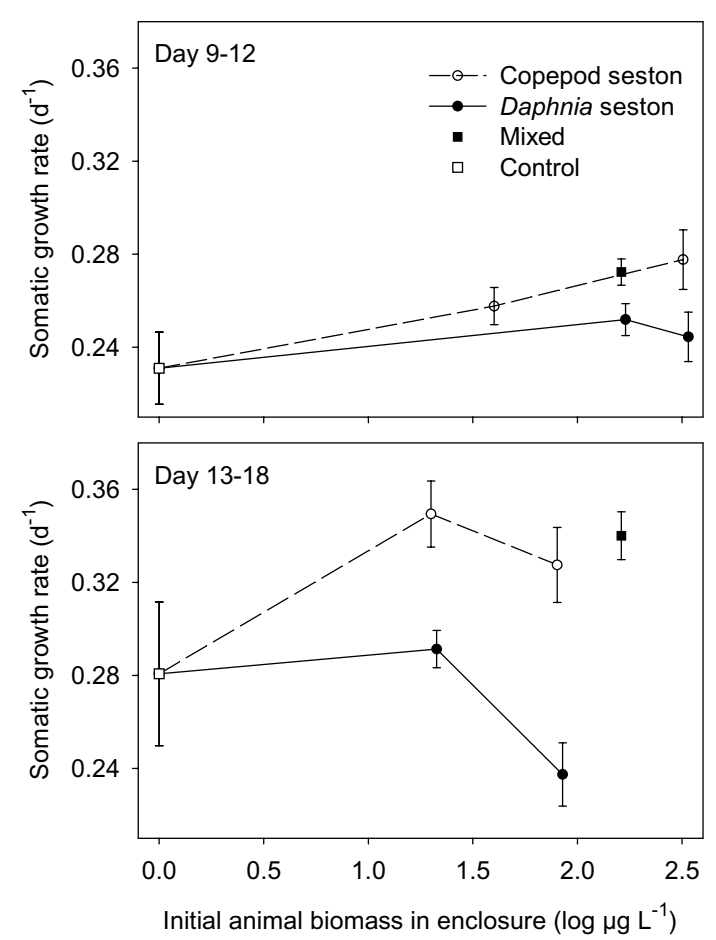

Figure 1. Somatic growth rate over three days for Daphnia magna fed seston from enclosures that were inoculated with different densities of zooplankton and which had been prehandled by the zooplankton community. Top panel: first period (days 9-12), bottom panel: second period (days 13-18). The enclosure seston used as experimental food, was at day zero enriched with copepods, daphniids, mixture of both copepods and daphniids or without zooplankton addition (control). Error bars denote \pm SE for five replicates. 
ment, which started 13 days after the zooplankton had been added to the enclosures, the results became clearer. Interestingly, overall growth of the daphniids was higher in the second experiment. The daphniids continued to grow faster on seston pre-handled by copepod grazers (ANOVA; $p<0.001$; Fig. 1 , bottom panel). We also observed that growth of $D$. magna declined with increasing densities of daphniids in the enclosures (ANOVA; Duncan's multiple range test; $p=0.0116$ ), which was not the case for the growth of the daphniids in the copepod gradient (ANOVA; Duncan's multiple range test; $p=0.2631$; Fig. 1). Post-hoc comparisons showed that in the second experiment, the copepod treatments were significantly different from both the control and the Daphnia treatments. In both experiments seston pre-treated by both grazers (mixed treatment) supported high Daphnia growth rates comparable to the growth on copepod water.

In contrast to what we found for Daphnia, we did not find significant differences in the developmental rate of the Eudiaptomus. The copepodites developed into higher stages but the development seemed to be independent of any pre-handling.

In the enclosures that were inoculated with Daphnia we established population growth rates between the different sampling dates. This was conducted over the last part of the experiment. The copepod densities did not change very much over this period. We found that Daphnia population growth rates were high with relatively large variations between the two replicate treatments in the period between days 13-16 (Fig. 2). In the following period (days 16-20) the growth rates declined somewhat but seemed to be independent of the initial densities. Only in the last period (days 20-24) we observed a significant decrease in growth rates relative to the period before (days 1620 versus days $20-24$; repeated measures analysis, $\left.F_{1.5}=8.27 ; p=0.0348\right)$. Moreover, in this period we also observed a stronger decrease in growth rates in those enclosures inoculated with the higher Daphnia densities.

When Daphnia somatic growth rates from the laboratory were compared with the population growth rates within the enclosures, we observed no significant correlation when the overlapping time intervals were used. However, since population growth is the numerical response of food quality

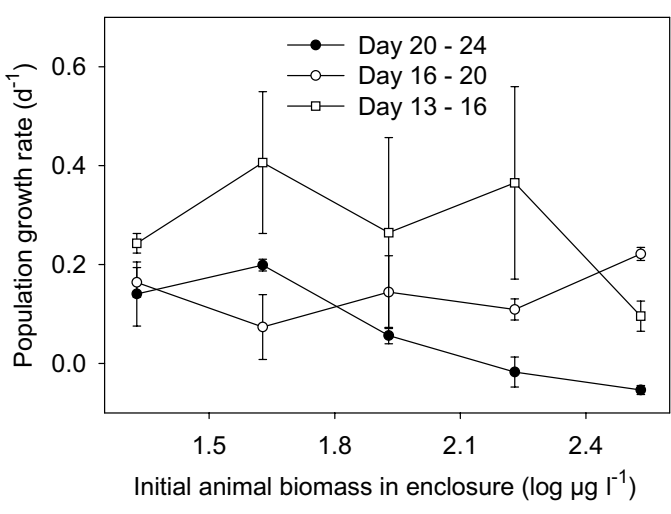

Figure 2. The impact of initial Daphnia densities on the population growth rates in the Daphnia enclosures, over three consecutive periods. Error bars denote $\pm \mathrm{SE}$ from 2 replicate enclosures.

changes, we expected the visible response to be delayed about one egg production time. This was indeed the case, and, the somatic growth was positively correlated with the population growth from the consecutive period $(y=0.65 x-0.058$; $r_{2}=0.15 ; p=0.020$ ).

During the two laboratory experiments the animals faced different amounts of food in terms of carbon (Fig. 3), with generally higher values in the first experiment. However, only in the first experiment there was significantly lower carbon in the Daphnia treatment (ANOVA; $p=0.0098$ ) compared to the copepod treatment. The content of particulate nitrogen and phosphorus followed a similar pattern to carbon.

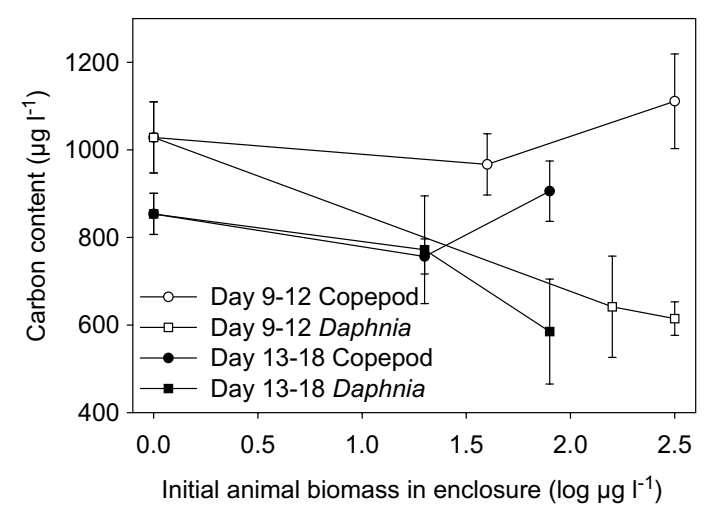

Figure 3. The average particulate carbon content $(<100 \mu \mathrm{m})$ during two laboratory experiments, conducted between days 912 and $13-18$. Error bars are \pm SE from two respectively three measurements. 
At the end of the enclosure experiment the total fatty acid content per litre of the seston in the Daphnia treatments was considerably lower than in the copepod treatments, and also the content of the essential $\omega 3$ fatty acids at days 20 and 24 were significantly affected by the type of grazer (Daphnia versus copepod; repeated measures analysis, $\left.F_{1.9}=31.8 ; p<0.001\right)$. When the content of the $\omega 3$ fatty acids of the animals was studied we found the reverse pattern. Copepods (mixtures of both calanoids and cyclopoids) had a significantly lower content of these fatty acids compared to daphniids (ANOVA; $p=0.039$ ), but a higher content of $\omega 6$ fatty acids. Moreover, generally the copepods had lower concentrations of the longer fatty acids (Table 1). We computed the $\omega 3: \omega 6$ ratio of both

Table 1 . The average fatty acid content ( $\left.\mu \mathrm{g} \mathrm{mg} \mathrm{dry} \mathrm{weight}{ }^{-1}\right)$ and SE (two measures, one for each replicate enclosure) for copepods and Daphnia at the end of the enclosure experiment (day 24)

\begin{tabular}{|c|c|c|c|c|}
\hline \multirow[t]{2}{*}{ Parameters } & \multicolumn{2}{|l|}{ Daphnia } & \multicolumn{2}{|l|}{ Copepods } \\
\hline & $\mu \mathrm{g} \mathrm{mg} \mathrm{DW}^{-1}$ & $\mathrm{SE}$ & $\mu \mathrm{g} \mathrm{mg} \mathrm{DW}^{-1}$ & SE \\
\hline $14: 0$ & 10.09 & 2.81 & 8.90 & 1.38 \\
\hline $16: 0$ & 18.25 & 7.33 & 20.04 & 2.99 \\
\hline $16: 1 \omega 7$ & 2.30 & 0.19 & 0.30 & 0.16 \\
\hline 18:0 & 15.28 & 8.63 & 5.41 & 1.63 \\
\hline $18: 1 \omega 12 / \omega 9$ & 5.77 & 1.88 & 4.49 & 3.67 \\
\hline $18: 1 \omega 7$ & 2.01 & 0.48 & 1.35 & 0.88 \\
\hline $18: 2 \omega 6$ & 7.83 & 0.24 & 1.67 & 1.12 \\
\hline $18: 3 \omega 6$ & 0.55 & 0.01 & 0.30 & 0.15 \\
\hline $18: 3 \omega 3$ & 5.09 & 0.72 & 0.71 & 0.36 \\
\hline $18: 4 \omega 3$ & 4.08 & 0.35 & 0.90 & 0.64 \\
\hline $20: 00$ & 0.64 & 0.17 & & \\
\hline $20: 1 \omega 7$ & 0.11 & 0.03 & 0.39 & 0.10 \\
\hline $20: 2 \omega 6$ & 0.85 & 0.03 & & \\
\hline $20: 3 \omega 6$ & 0.30 & 0.03 & & \\
\hline $20: 4 \omega 6$ & 8.87 & 0.00 & 2.50 & 1.81 \\
\hline $20: 3 \omega 3$ & 0.14 & 0.03 & & \\
\hline $20: 4 \omega 3$ & 1.01 & 0.03 & & \\
\hline $20: 5 \omega 3$ & 9.09 & 0.17 & 2.31 & 1.75 \\
\hline $22: 0$ & 0.37 & 0.14 & & \\
\hline $22: 2 \omega 6$ & 0.09 & 0.09 & & \\
\hline $22: 4 \omega 6$ & 0.09 & 0.09 & & \\
\hline $22: 5 \omega 3$ & 1.18 & 0.22 & & \\
\hline $22: 6 \omega 3$ & 9.90 & 0.03 & & \\
\hline $24: 0$ & 0.63 & 0.34 & & \\
\hline $24: 1 \omega 9$ & 0.73 & 0.12 & & \\
\hline
\end{tabular}

animals and seston, a measure often used to define food quality of zooplankton, with higher ratios normally considered to represent higher quality. As expected, there were differences between zooplankton taxa, with daphniids having higher $\omega 3: \omega 6$ ratios than copepods (Fig. 4; ANOVA; $p<0.001$ ), but no significant differences between animals taken from different densities. The $\omega 3: \omega 6$ ratios in the seston did not seem to vary much between the enclosures, only in the highest copepod density the $\omega 3: \omega 6$ ratios were elevated. This was due to the small amounts of both fatty acid families in this treatment. Thus, we found that seston pre-handled by copepods had significantly higher $\omega 3: \omega 6$ ratios than seston pre-handled by daphniids (Daphnia versus copepod; repeated measures analysis over the last two sampling dates, $p=0.006$; Fig. 4.).

Neither carbon, nitrogen or phosphorus showed a strong correlation with Daphnia growth in our laboratory study. The strongest positive correlation with Daphnia somatic growth and food quantity aspects was with the concentration of 20:4 $\omega 3$. At higher concentrations the animals showed significantly higher growth rates (Fig. 5; $\left.y=0.171 x+0.21, r^{2}=0.37, p<0.001\right)$. In a backward stepwise multiple regression with D. magna somatic growth as the dependent variable, $54 \%$ of the variance was explained by the content of $20: 4 \omega 6,20: 4 \omega 3$ and 20:5 $\omega 3$. On the other hand, in the enclosures the population growth rates during the last growth period (days

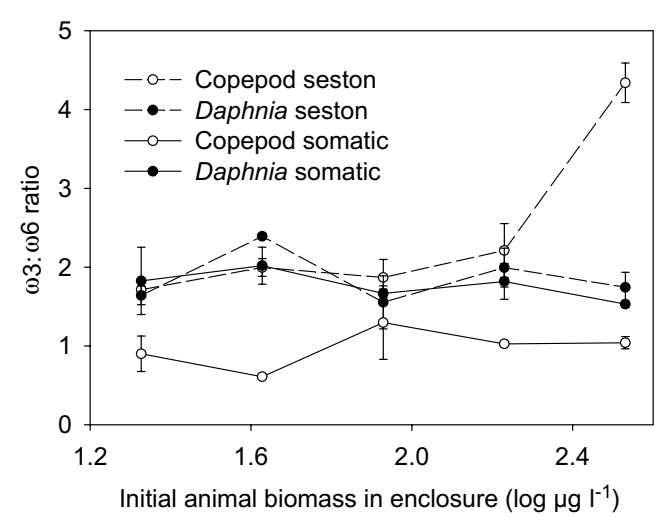

Figure 4. The $\omega 3: \omega 6$ ratio of enclosure seston and zooplankton at the end of the enclosure experiment (day 24). The seston values are the averages from the two last samplings (days 20 and 24). Error bars are $\pm \mathrm{SE}$ from two replicate enclosures. 


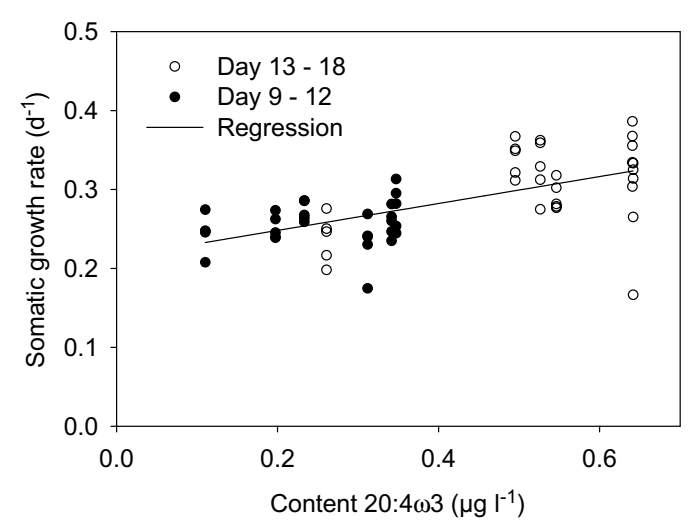

Figure 5. The correlation between somatic growth rates for Daphnia magna and the average content of eicosatetraenoic acid $(20: 4 \omega 3)$ in the food. The daphniids were fed seston from enclosures that were inoculated with different densities of zooplankton. The enclosure seston was pre-handled by the zooplankton community over either $9(\bullet)$ or $13(\bigcirc)$ day period. The regression equation is $y=0.171 x+0.21, r^{2}=0.37, p<0.001$.

20-24) correlated most strongly with the particulate nitrogen content per litre (Fig. 6; $y=0.0040$ $\left.x-0.184 ; r^{2}=0.78 ; p<0.001\right)$.

We calculated the ratios of the different fatty acids to carbon as a measure of food quality rather than quantity, and computed the correlations of the different parameters with both somatic and population Daphnia growth. In the laboratory experiment several of the factors had a positive correlation with somatic growth. Neither P:C nor $\mathrm{N}: \mathrm{C}$ had a significant impact on growth, but sev-

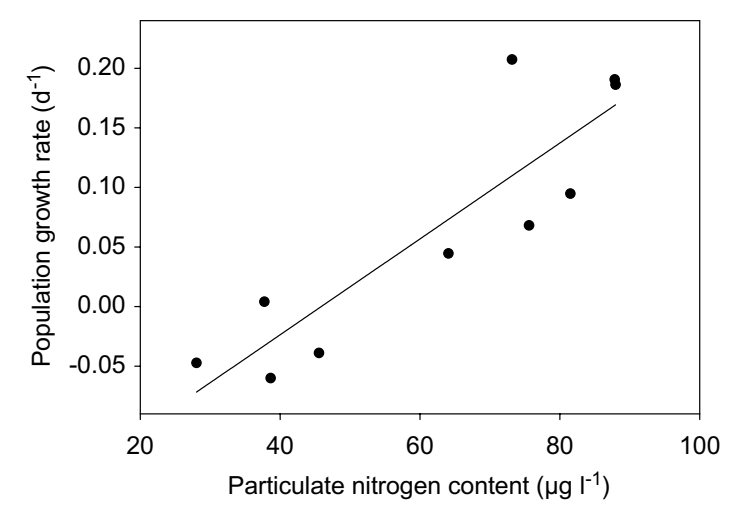

Figure 6. The correlation between population growth rate and the particulate nitrogen content. The population growth between days 20-24 in correlation with the particulate nitrogen content from day 22. The regression equation is $y=0.0040 x-0.184, r^{2}=0.78, p=0.001$.
Table 2. Correlation between seston quality parameters and Daphnia growth in laboratory (Somatic) and in enclosures (Population)

\begin{tabular}{|c|c|c|c|c|}
\hline \multirow{2}{*}{ Parameters } & \multicolumn{2}{|c|}{ Somatic growth, $g$} & \multicolumn{2}{|c|}{ Population growth, $r$} \\
\hline & $r^{2}$ & $p$ & $r^{2}$ & $p$ \\
\hline $\mathrm{P}: \mathrm{C}$ & 0.17 & $<0.001$ & 0.30 & 0.10 \\
\hline $\mathrm{N}: \mathrm{C}$ & 0.19 & $<0.001$ & 0.51 & 0.021 \\
\hline 16:0 & 0.16 & 0.0014 & 0.10 & 0.38 \\
\hline $16: 1 \omega 7$ & 0.19 & $<0.001$ & 0.38 & 0.054 \\
\hline $18: 0$ & 0.0004 & 0.87 & 0.23 & 0.15 \\
\hline $18: 1 \omega 12 / \omega 9$ & 0.16 & 0.002 & 0.026 & 0.65 \\
\hline $18: 1 \omega 7$ & 0.17 & 0.001 & 0.21 & 0.17 \\
\hline $18: 2 \omega 6$ & 0.01 & 0.36 & 0.14 & 0.28 \\
\hline $18: 3 \omega 6$ & 0.06 & 0.05 & 0.0022 & 0.90 \\
\hline $18: 3 \omega 3$ & 0.004 & 0.63 & 0.0095 & 0.79 \\
\hline $18: 4 \omega 3$ & 0.06 & 0.05 & 0.14 & 0.27 \\
\hline 20:0 & 0.13 & 0.01 & 0.12 & 0.57 \\
\hline $20: 1 \omega 7$ & 0.01 & 0.64 & 0.07 & 0.72 \\
\hline $20: 2 \omega 6$ & 0.04 & 0.15 & 0.05 & 0.77 \\
\hline $20: 3 \omega 6$ & 0.03 & 0.21 & nd & \\
\hline $20: 4 \omega 6$ & 0.29 & $<0.001$ & 0.14 & 0.28 \\
\hline $20: 3 \omega 3$ & 0.03 & 0.17 & 0.096 & 0.50 \\
\hline $20: 4 \omega 3$ & 0.33 & $<0.001$ & 0.008 & 0.81 \\
\hline $20: 5 \omega 3$ & 0.22 & $<0.001$ & 0.28 & 0.11 \\
\hline $22: 6 \omega 3$ & 0.10 & 0.014 & 0.22 & 0.17 \\
\hline 24:0 & 0.20 & 0.001 & 0.006 & 0.92 \\
\hline
\end{tabular}

In all correlations the ratio of the specific compound to carbon is used (e.g. fatty acid:C or N:C).

eral of the fatty acids seemed to be important. Again, 20:4 $\omega 3$ showed the strongest correlation with somatic growth (Table 2). In a backward stepwise multiple regression with Daphnia somatic growth as the dependent variable $52 \%$ of the variance was explained by the ratio of $18: 3 \omega 3$ and $20: 4 \omega 6$ to the total carbon. The population growth was only positively related to the $\mathrm{N}: \mathrm{C}$ ratio (Table 2).

\section{Discussion}

A few studies have shown that herbivorous copepods and daphniids have different impacts on phytoplankton communities. In a laboratory experiment, Rothhaupt (1997) showed that different algae became dominant when different grazers were present. Whereas small sized algae became 
dominating in a system with Eudiaptomus, larger algae prevailed under a regime of Daphnia grazing. Sommer et al. (2001) reported a similar result from an enclosure study comparing grazing effects of daphniids and copepods on the phytoplankton community. They found a strong impact on the size structure of the phytoplankton community dependent on the different zooplankton taxa. These differences can be linked to the different feeding mode of copepods and cladocerans. Many studies have described that copepods can detect and select favourable particles (e.g. DeMott, 1986, Butler et al., 1989). Daphniids on the other hand feed unselectively, but are able to reject unsuitable particles (DeMott, 1982; Kerfoot \& Kirk, 1991). There are several explanations for the increase in smaller particles under copepod dominance: the copepods primarily feed on the larger algae, thus leaving more nutrients available for the smallersized algae to grow. Alternatively, it could be the case that with copepod grazing, nutrients are released in the water column, which can rapidly be taken up by small-size phytoplankton biomass with their shorter generation times and larger surface to volume ratios. A third possible explanation is that the effect is indirect via the microzooplankton. Copepods also feed on ciliates, and the densities of these potential algal grazers on the smaller phytoplankton species decreases as a result of the presence of copepods (Zöllner et al., submitted).

We found Daphnia growth to be strongly influenced by the pre-treatment of the seston community by different guilds (Fig. 1), and observed a positive effect of copepod pre-handling on growth. Our experiments were conducted during a spring bloom, in which the seston community already largely consists of smaller sized algae (Sommer et al., 1986). However, the copepod grazing still had a positive impact on Daphnia growth. In phytoplankton counts (from day 11) of this enclosure experiment, we indeed observed that the smaller algae $(<100 \mu \mathrm{m})$ decreased with Daphnia density, while no effect was visible for the copepods (Feuchtmayr, unpublished results). In contrast to the effects observed for Daphnia growth, we observed no differences in the development of the copepodites grown under different conditions. This could be interpreted as no impact of Daphnia grazing on the food for copepods, but it could well be the case that we did not detect any differences since we used too blunt a technique, determining development in a mixture of copepodite stages over 5 days. Preferably in such a short interval, we should have used more sensitive methods such as egg production experiments or RNA/DNA measurements (Saiz et al., 1998; Vrede et al., 2002). Therefore we can not draw any real conclusions from this part of the experiment.

Previous studies have tried to link somatic growth rates of Daphnia with seston characteristics in a range of different lakes (Müller-Navarra, 1995; Elser et al., 2001; Wacker \& von Elert, 2001). In Schöhsee, Daphnia growth over an entire season was closely linked to the content of the polyunsaturated fatty acid EPA (20:5 13 ) (Müller-Navarra, 1995). In a similar study in Lake Constance the content of $\alpha$-linolenic acid $(18: 3 \omega 3)$ was the strongest factor correlating with Daphnia growth (Wacker \& von Elert, 2001). These different fatty acids are members of the $\omega 3$ fatty acid family, which are essential for most consumers. However, since many consumers have the possibility to desaturate and elongate the fatty acids on other positions (Olsen, 1999), they are not totally dependent on the content of a single $\omega 3$ fatty acid, although the conversion of the fatty acid may be slow (von Elert, 2002). In this study we also found that somatic growth of Daphnia was positively related to the content of a few polyunsaturated fatty acids. The strongest single regression was with the absolute concentration of $20: 4 \omega 3$. This fatty acid is similar to EPA, differing only in one (non-essential) double bond. It is often found in low concentrations and therefore seldom mentioned in other studies. Both EPA and 20:4 $1 \omega 6$ also correlated positively to our measures of growth, not only in absolute concentrations, but also their ratio to carbon (Table 2). Nevertheless, this is correlative evidence for the importance of certain fatty acids, and it cannot be ruled out that a different factor correlating with the fatty acids is in fact the quality determining factor (Boersma \& Kreutzer, 2002; Becker \& Boersma, 2003).

In the Daphnia enclosures, the population growth showed considerable fluctuations, with a general decrease over the last sampling intervals (Fig. 2). Surprisingly, the growth of the populations in the enclosures seemed to be independent of the initial animal biomass between days 13-16 and 16-20, which, if indeed the daphniids exerted 
the predation pressure on the phytoplankton as suggested, is difficult to explain. Only in the interval between days 20 and 24, we observed a decline of population growth rate with density. The patterns found for the somatic growth rates in the laboratory and the population growth rates in the enclosures were similar, although not significantly correlated when the same time period was chosen for both measures. The correlation of the somatic growth rates in the laboratory with the population growth rate in the period immediately afterwards was, however, significant. This is understandable since the population growth in our study was a numerical response. Therefore we would expect that the response due to food changes could be seen not earlier as one egg production cycle later than the food effect.

Surprisingly, we did observe different correlations of food quality parameters between laboratory growth and population growth in the enclosures, even though the two growth measurements are correlated. From the laboratory microcosms we would infer that several fatty acids are limiting, whereas in the enclosures we would conclude that the $\mathrm{N}: \mathrm{C}$ ratio was the most important limiting factor. From these results an interesting question arises: are results linking growth differences to food quality in laboratory comparable to what happens in the field? Microcosm experiments have been used in several studies to investigate food quality limitations (e.g. Müller-Navarra, 1995; Boersma et al., 2001; Elser et al., 2001; Wacker \& von Elert, 2001). The method is sensitive, easy to perform and needs only a few days. Moreover, it is closely linked to population growth in the laboratory (Lampert \& Trubetskova, 1996). Our results here, however, indicate that we might be measuring two different things. Of course, under normal field conditions potential population growth rates as measured in the laboratory will never be attained because predation is present in the field, a factor largely excluded in our enclosures. The most straightforward explanation of the differences in results is that in a correlation analysis one factor has to show the highest correlation, the exact nature of which might be coincidental. This might be true, but does not explain our observation that only $\mathrm{N}: \mathrm{C}$ ratio correlates significantly with population growth, and many other factors correlate with somatic growth. Where establishing population growth rates under (semi-) natural conditions has the advantage that one does not have to take the water into the laboratory with possible changes the nature of the seston, it has the disadvantage that variability in estimates of population growth rates is usually high. In contrast to somatic growth estimates showing direct responses (weight increase), numerical responses involve several more factors. Where somatic growth rates only include somatic growth of juvenile animals the population growth rates also include egg production, moulting success etc, and it could well be the case that different factors are of important in different life-stages (see also Becker \& Boersma, 2003). Interestingly enough, in a previous enclosure study on the effect of fatty acid additions on population growth of Daphnia also no effect was found of the addition of emulsions of highly unsaturated fatty acids (Boersma \& Stelzer, 2000). These differences in results between laboratory studies and field observations certainly warrant future investigations.

At the end of the enclosure experiments (days 20 and 24) we observed distinct differences in the fatty acid concentration per litre of the different treatments, with lower fatty acid content in the Daphnia seston. In the animals we found the opposite, with higher concentrations of both $\omega 3$ and $\omega 6$ fatty acids in the daphniids than in the copepods. There were also differences in the fatty acid composition between the two taxa, and especially the long chained fatty acids were found in much lower concentrations in the copepods (Table 1). Interestingly, even though the $\omega 3: \omega 6$ ratio of the seston were similar in the enclosures (only the highest copepod density differed), the ratio between $\omega 3: \omega 6$ fatty acids was significantly higher in the daphniids than in the copepods (Fig. 4). It appears as if these ratios were stabile as it did not decrease with increasing animal densities, and also not during less optimal conditions when growth was retarded (Fig. 2; last period), which suggests some kind of homeostasis for fatty acids (see also Anderson \& Pond, 2000). These differences have also been found in laboratory experiments. In two separate studies copepods and daphniids were cultured with Cryptomonas sp., and the fatty acid pattern of each zooplankton was determined. Daphniids showed a high $\omega 3: \omega 6$ ratio of 12.8 (from Weers et al., 1997) and Eudiaptomus 
a lower $\omega 3: \omega 6$ ratio 5.8 (from von Elert \& Stampfl, 2000). These patterns suggest different requirements for different fatty acids between copepods and cladocerans (Boersma \& Stelzer, 2000), which could have as a consequence that different fatty acids are left in the seston in a similar way that macronutrients are recycled differently by cladocerans and copepods (Rothhaupt, 1997), although it seems to be the case that the daphniids with their non-selective feeding mode reflect the seston much more than the copepods.

Daphniids in high densities had a negative impact on Daphnia growth. However, the question is whether the presence of copepods is really beneficial to the daphniids, or whether the absence of Daphnia is the more important factor. In that case the control treatments (without copepods and daphniids) should yield the same growth as the ones with copepods. This was clearly not the case. In the first experiment the control supported low growth rates and the effect of copepods on the growth rate was marginally significant (ANOVA; $p=0.06$ ). In the second experiment the growth of the control was on an intermediate level between the copepod and Daphnia treatments, and significantly different from the copepod treatments. Interestingly, growth of Daphnia on the mixedtreatments was relatively high even though we had an initial Daphnia density of 5 ind $1^{-1}$. The probable reason for this was that the Daphnia development in this treatment was much slower compared to only-Daphnia treatment. The effective Daphnia density development in the mixed treatment was more similar to the lowest Daphnia treatment. Most likely, the slower development in this treatment was caused by the higher predation pressure of the cyclopoid copepods in those enclosures.

We conclude that the presence of copepods indeed increased the feeding conditions for Daphnia. Even though our copepod growth data were too weak to state in which direction the interaction would have affected the copepods, there is a good chance that the interaction is beneficial for both organisms, caused by the different feeding behaviours with preferences for differently sized particles. Our experiment was conducted during spring conditions where the seston community consists largely of small particles. It is interesting that already under these conditions we can detect differences between which zooplankton taxa that have pre-handled the seston. This effect should probably even stronger still in late summer when larger particles and colonies are more common in the seston.

\section{Acknowledgements}

This work would not have been possible without the help of all of the members of the IfM-MPIL food web team. Frank Sommer and Eckart Zöllner are especially acknowledged. Winfried Lampert and Ulrich Sommer are thanked for their continuous support. We thank Heinke Buhtz and Dieter Albrecht for laboratory assistance. Lena Sivars is thanked for stimulating discussions and comments on earlier drafts of this manuscript. This research was supported by the DFG Grants: BO 1488/3-1, SA 638/1-1 and SO 145/19-1.

\section{References}

Anderson, T. R. \& D. W. Pond, 2000. Stoichiometric theory extended to micronutrients: comparison of the roles of essential fatty acids, carbon, and nitrogen in the nutrition of marine copepods. Limnology and Oceanography 45: 11621167.

Becker, C. \& M. Boersma, 2003. Resource quality effects on life histories in Daphnia. Limnology and Oceanography 48: 700706.

Bengtsson, J., 1986. Life histories and interspecific competition between three Daphnia species in rockpools. Journal of Animal Ecology 55: 641-655.

Boersma, M., 1995. Competition in natural populations of Daphnia. Oecologia 103: 309-318.

Boersma, M., 2000. The nutritional quality of P-limited algae for Daphnia. Limnology and Oceanography 45: 1157-1161.

Boersma, M. \& C. Kreutzer, 2002. Life at the edge: is food quality really of minor importance at low quantities? Ecology 83: 2552-2561.

Boersma, M., C. Schöps \& E. Mccauley, 2001. Nutritional quality of seston for the freshwater herbivore Daphnia galeata $x$ hyalina: biochemical versus mineral limitations? Oecologia 129: 342-348.

Boersma, M. \& C. -P. Stelzer, 2000. Response of a zooplankton community to the addition of unsaturated fatty acids: an enclosure study. Freshwater Biology 45: 179-188.

Butler, N. M., C. A. Suttle \& W. E. Neill, 1989. Discrimination by freshwater zooplankton between single algal cells differing in nutritional status. Oecologia 78: 368-372.

Cowles, T. J., R. J. Olson \& S. W. Chisholm, 1988. Food selection by copepods discrimination on the basis food quality. Marine Biology 100: 41-50.

Cuddington, K. M. \& E. Mccauley, 1994. Food-dependent aggregation and mobility of the water fleas Ceriodaphnia dubia and Daphnia pulex. Canadian Journal of Zoology 72: 1217-1226. 
DeMott, W. R., 1982. Feeding selectivities and relative Ingestion Rates of Daphnia and Bosmina. Limnology and Oceanography 27: 518-527.

DeMott, W. R., 1986. The role of taste in food selection by freshwater zooplankton. Oecologia 69: 334-340.

DeMott, W. R. \& W. C. Kerfoot, 1982. Competition among cladocerans: nature of the interaction between Bosmina and Daphnia. Ecology 63: 1949-1966.

DeMott, W. R. \& F. Moxter, 1991. Foraging on cyanobacteria by copepods - responses to chemical defenses and resource abundance. Ecology 72: 1820-1834.

Elser, J. J., K. Hayakawa \& J. Urabe, 2001. Nutrient limitation reduces food quality for zooplankton: Daphnia response to seston phosphorus enrichment. Ecology 82: 898-903.

Flöder, S. \& U. Sommer, 1999. Diversity in planktonic communities: an experimental test of the intermediate disturbance hypothesis. Limnology and Oceanography 44: 1114-1119.

Gliwicz, Z. M., 1994. Retarded growth of cladoceran zooplankton in the presence of a copepod predator. Oecologia 97: 458-461.

Gliwicz, Z. M. \& G. Umana, 1994. Cladoceran body size and vulnerability to copepod predation. Limnology and Oceanography 39: 419-424.

Grasshoff, K., M. Ehrhardt \& K. Kremling, 1983. Methods of Seawater Analysis, 2nd edn. Verlag Chemie.

Hessen, D. O., 1990. Niche overlap between herbivorous cladocerans; the role of food quality and habitat homogeneity. Hydrobiologia 190: 61-78.

Hessen, D. O. \& A. Lyche, 1991. Interspecific and intraspecific variations in zooplankton element composition. Archiv Fur Hydrobiologie 121: 343-353.

Huisman, J. \& F. J. Weissing, 1999. Biodiversity of plankton by species oscillations and chaos. Nature 402: 407-410.

Hutchinson, G. E., 1961. The paradox of the plankton. American Naturalist 95: 137-145.

Interlandi, S. J. \& S. S. Kilham, 2001. Limiting resources and the regulation of diversity in phytoplankton communities. Ecology 82: 1270-1282.

Jensen, K. H., P. Larsson \& G. Hogstedt, 2001. Detecting food search in Daphnia in the field. Limnology and Oceanography 46: 1013-1020.

Jürgens, K., S. A. Wickham, K. O. Rothhaupt \& B. Santer, 1996. Feeding rates of macro- and microzooplankton on heterotrophic nanoflagellates. Limnology and Oceanography 41: 1833-1839.

Kerfoot, W. C., 1977. Implications of copepod predation. Limnology and Oceanography 22: 316-325.

Kerfoot, W. C. \& K. L. Kirk, 1991. Degree of taste discrimination among suspension-feeding cladocerans and copepods - Implications for detritivory and herbivory. Limnology and Oceanography 36: 1107-1123.

Lampert, W., 1987. Feeding and nutrition in Daphnia. Memorie dell'Istituto Italiano di Idrobiologia 45: 143-192.

Lampert, W. \& I. Trubetskova, 1996. Juvenile growth rate as a measure of fitness in Daphnia. Functional Ecology 10: 631-635.

Matveev, V. F., 1983. Estimating competition in cladocerans using data on dynamics of clutch size and population density. Internationale Revue der Gesamten Hydrobiologie 68: 785-798.
Müller-Navarra, D., 1995. Evidence that a highly unsaturated fatty acid limits Daphnia growth in nature. Archiv Für Hydrobiologie 132: 297-307.

Olsen, Y., 1999, Lipids and essential fatty acids in aquatic food webs: what can freshwater ecologists learn from mariculture? In Arts, M. T. \& B. C. Wainman (eds), Lipids in Freshwater Ecosystems, Springer-Verlag, New York, Inc: 161-202.

Rothhaupt, K. O., 1997. Grazing and nutrient influences of Daphnia and Eudiaptomus on phytoplankton in laboratory microcosms. Journal of Plankton Research 19: 125-139.

Saiz, E., A. Calbet, A. Fara \& E. Berdalet, 1998. RNA content of copepods as a tool for determining adult growth rates in the field. Limnology and Oceanography 43: 465-470.

Santer, B. \& F. van den Bosch, 1994. Herbivorous nutrition of Cyclops vicinus - the effect of a pure algal diet on feeding, development, reproduction and life-cycle. Journal of Plankton Research 16: 171-195.

Sommer, U., Z. M. Gliwicz, W. Lampert \& A. Duncan, 1986. The PEG model of seasonal succession of planktonic events in fresh waters. Archiv Für Hydrobiologie 106: 433-471.

Sommer, U., F. Sommer, B. Santer, C. Jamieson, M. Boersma, C. Becker \& T. Hansen, 2001. Complementary impact of copepods and cladocerans on phytoplankton. Ecology Letters 4: 545-550.

Sommer, U., F. Sommer, B. Santer, E. Zöllner, K. Jürgens, C Jamieson, M. Boersma \& K. Gocke, 2003. Daphnia versus copepod impact on summer phytoplankton: functional compensation at both trophic levels. Oecologia, DOI 10.1007/s00442-003-1214-7.

Tilman, D., 1982. Resource competition and community structure. Princeton University Press, Princeton, NJ.

Vanni, M. J., 1986. Competition in zooplankton communities: suppression of small species by Daphnia pulex. Limnology and Oceanography 31: 1039-1056.

Villar-Argaiz, M. \& R. W. Sterner, 2002. Life history bottlenecks in Diaptomus clavipes induced by phosphorus-limited algae. Limnology and Oceanography 47: 1229-1233.

von Elert, E., 2002. Determination of limiting polyunsaturated fatty acids in Daphnia galeata using a new method to enrich food algae with single fatty acids. Limnology and Oceanography 47: 1764-1773.

von Elert, E. \& P. Stampfl, 2000. Food quality for Eudiaptomus gracilis: the importance of particular highly unsaturated fatty acids. Freshwater Biology 45: 189-200.

Vrede, T., J. Persson \& G. Aronsen, 2002. The influence of food quality (P:C ratio) on RNA:DNA ratio and somatic growth rate of Daphnia. Limnology and Oceanography 47: 487-494.

Wacker, A. \& E. von Elert, 2001. Polyunsaturated fatty acids: evidence for non-substitutable biochemical resources in Daphnia galeata. Ecology 82: 2507-2520.

Weers, P. M. M., K. Siewertsen \& R. D. Gulati, 1997. Is the fatty acid composition of Daphnia galeata determined by the fatty acid composition of the ingested diet? Freshwater Biology 38: 731-738.

Wiltshire, K. H., M. Boersma, A. Möller \& H. Buhtz, 2000. Extraction of pigments and fatty acids from the green alga Scenedesmus obliquus (Chlorophyceae). Aquatic Ecology 34: 119-126. 